Voix et Images

vorxetimages

\title{
Le Cas C « Le Cassé " sur le tchesteurfilde, un cas pour les psychanalystes [dit-il] ou d'une esthétique du bâton, S-T-Tick : stick [dit-il]
}

Henri-Paul Jacques

Volume 4, numéro 2, décembre 1978

Guy Lafond

URI : https://id.erudit.org/iderudit/200159ar

DOI : https://doi.org/10.7202/200159ar

Aller au sommaire du numéro

Éditeur(s)

Les Presses de l'Université du Québec

ISSN

0318-9201 (imprimé)

1705-933X (numérique)

Découvrir la revue

Citer cet article

Jacques, H.-P. (1978). Le Cas C « Le Cassé " sur le tchesteurfilde, un cas pour les psychanalystes [dit-il] ou d'une esthétique du bâton, S-T-Tick : stick [dit-il]. Voix et Images, 4(2), 300-318. https://doi.org/10.7202/200159ar d'utilisation que vous pouvez consulter en ligne. 


\title{
Le Cas C
}

«Le Cassé» sur le tchesteurfilde, un cas pour les psychanalystes [dit-il]

ou

\author{
d'une esthétique du bâton, S-T-Tick : stick [dit-il] \\ (DEUXIÈME TRANCHE')
}

(Dans la première tranche de cet essai, j'indiquais ma préférence pour le choix d'une démarche d'allure pédagogique et didactique, démarche que je conserverai dans la suite de mon analyse. Cependant, avant de reprendre mon propos où je l'avais laissé, je résumerai brièvement ce qui a été précédemment exposé tout en reproduisant la chapitration retenue :

A. Quelques thèmes du "Cassé"

1 - L'incipit et la stick-o-manie : «jeux de mains... "

"Cette chambre lui a coûté cinq piasses" (p. 13).

Quelques considérations sur l'importance de l'incipit en général et de cet incipit-ci en particulier.

Hypothèse provisoire à vérifier rigoureusement dans le texte: le chiffre cinq est un symbole universel possible de la masturbation par référence aux cinq doigts de la main.

Vérifications de l'hypothèse dans le texte:

(a) au niveau du taire l'inavouable

Relevé des récurrences obsédantes du chiffre cinq, du mot main(s), du mot doigt(s).

(b) au niveau de l'inter-taire/dire

Analyse de cinq passages du "Cassé», qui, d'une manière midite/mi-tue, réfèrent à la masturbation.

C'est là que, faute de temps et d'espace, je m'étais arrêté et c'est là que je reprendrai.

Mais pour mi-dire/mi-taire, je préciserai dès maintenant certains des sujets que j'ai l'intention de traiter, me réservant cependant le droit de modifier à mon gré l'ordre de présentation des sujets annoncés selon la logique interne de mon discours analytique.

(c) au niveau du dire expressément, transgression et subversion

(d) intermède méthodologique: le phénomène de la plurilmultiversion

(e) pause théorique : deux sortes de "secrets", un seul discours 
2-Le Surmoi et la stick-o-mani(e)pulation : “... jeux de vilains"

3-Ti-Jean et la stick-o-crassie des crasseux: une conception de la femme

4-Ah! Philo-men, Philo-main-e! Philomène et le sexe... dans les/des mots

5-Dents pour dans, face pour face : la loi du talion

6 - Stick-tige-TIGEANT : un univers trop grand pour le rejeton (pe)TI (t)-JEAN

7 - Libido/destrudo et la cassure du Cassé/Casseur : attaquer pour se défendre

8-?????

Quelques autres sujets dont j'ignore encore ce qu'ils seront et pour lesquels "le Cassé " me mènera, comme Ti-Jean lui-même est mené par ses propres fantasmes sur «j'aime (philô, en grec) Philomène ".

\section{B. La langue du Cassé}

1 - Selon les coups de "bâtons" à la Shakespeare: le "joual" comme langue maternelle

(a) le laid et le mal: le grand scandale des bourgeois

(b) le lait maternel et le bien : le petit confort des prolétaires

2 - Selon une typologie psychanalytique

(a) registre oral et bilinguisme: cunnilingue et/ ou fellation?

(b) registre anal et déplacement : sens devant derrière?

(c) registre phallique : le fendu defendu?

C. La générativité verbale et textuelle, "libri aut liberi "

1 -Les enfants des mots et du texte: enchaînements et mécanismes associatifs

(a) la séduction des mots par les mots : accouplements verbaux

(b) l'engendrement du texte par le texte: naissance du livre

2- La communication parallèle et la communication préalable

(a) la para-communication

(b) la pré-communication

\section{Conclusion}

1 - Les Carnets de Lénine et le Surréalisme

2-Pauvre Reich! lui aussi il a fini par crever

3 - Lire «le Cassé »

Je reprends donc la suite de ma première tranche.)

(c) au niveau du dire expressément

Je me suis imposé de vérifier une hypothèse de départ, établie sur l'incipit du «Cassé », à savoir que le chiffre cinq est un symbole universel possible de la masturbation par référence aux cinq doigts de la main. J'ai tenté de démontrer l'actualisation de ce symbole dans le texte analysé et j'ai traduit en clair quelques passages qui, malgré leur expression dans un langage hermétique, font eux aussi allusion à la masturbation. Traduc- 
tion : ici, transposition du code de l'inconscient dans le code du conscient. Je citerai, pour ceux qui n'ont pas encore lu "le Cassé " ou qui n'ont pas procédé d'eux-mêmes aux mises en rapport obligées, les passages qui confirment cette hypothèse.

Ti-Jean a loué une chambre à "cinq piasses" et il a téléphoné à Philomène de l'y rejoindre. Philomène réagit par un monologue intérieur: "cinq piasses. C'est pas vargeux, ça mon Ti-Jean» (p. 16) ; vargeux, adjectivation du mot "verge", légèrement déformé, dans tous ses sens (et synonyme, en particulier, du «stick/bâton» de Renaud ${ }^{2}$ ); expression tirée du registre phallique, qui se transposerait facilement en "y a rien là" dans le même registre, en "ça fesse pas fort/ça écrase rien" dans le registre anal et en "ça vaut pas une spitoune/y a pas d'quoi se décrocher la mâchoire" dans le registre oral (digression intentionnelle pour laisser entendre déjà ce que sera l'analyse de la langue du «Cassé» selon la typologie psychanalytique annoncée). Pour rejoindre Ti-Jean, «Philomène a marché jusqu'à l'avenue du Parc», puis «elle a commencé à faire du pouce ${ }^{3}$. II n'y a pas loin du pouce à toute la main, au chiffre cinq et à son multiple dix, à la confirmation explicite de l'hypothèse :

Au bout de dix minutes, une petite volkswageune a stoppé. Philomène a couru vers l'auto. Une main de femme a ouvert la portière.

$[\ldots]$

L'auto a démarré. Philomène est assise tout près d'une jeune femme d'environ vingt-cinq ans. Philomène pense qu'elle aurait préféré un homme. C'est plus agréable. On les attendrit. On leur arrache un petit cinq, un petit dix. $Y$ sont pas toujours beaux... mais ça fait rien... on peut pas tout avoir... quand y sont vieux, y tripotent pas... y s'contentent de la main. [P. 16-17. C'est moi qui souligne et qui ajoute les majuscules.]

Est-ce assez clair? Collocation de tous les éléments d'un ensemble dont chacun a été analysé séparément dans la première tranche de cet essai (y compris "l'auto" que je n'ai pas souligné pour respecter l'intelligence du lecteur qui a sans doute compris) : collocation, autre critère de repérage d'une émergence possible de l'inconscient et en même temps, dans des passages tels que celui-ci, confirmation de la validité de l'interprétation ${ }^{4}$. De plus, comme s'il ne suffisait pas de cette collocation de symboles, d'allusions, de cette mention explicite de la masturbation ( «y s'contentent de la $\operatorname{main}^{5} \%$ ), insertion dans tout cela d'un autre élément itératif convergent, "on leur arrache un petit cinq, un petit dix"; encore un cliché apparemment innocent, à l'air si peu «vargeux" qu'il risque de passer totalement inaperçu. Le sens de ce cliché est, dans ce contexte sexuel ${ }^{6}$, tellement symbolique - c'est-à-dire si loin de la conscience - qu'il exige une traduction:

II y a dans nos pays une expression grossière pour exprimer la masturbation : sich einen ausreissen (litt. s'en arracher un), ou sich einen herunterreissen (litt. s'en faire tomber un). Je ne saurais dire d'où viennent ces expressions, quelle image est au fond?. 
Le latin fournit la même «image" avec mascarpio/ «masturbateur", composé de mas (soit "mâle», soit déformation de manus/«main") et de carpo/ "cueillir-arracher ${ }^{8}$. Pour ceux qui abhorrent le nom de Freud et les comparaisons interlinguistiques, retournons à la «littérature" de langue française :

A ma vue, la femme, nue sur le lit, magnifique, se mettait à rire. [...] les yeux seuls vivant dans son visage immobile de plaisir, passant sans répit de mon visage à mon sexe que ma main travaillait avec tout le désir que j'avais pour elle, elle me parla, me supplia, m'ordonna [...] Fais-le fort maintenant sois gentil fais-le fort arrache-to $i$ arrache-toi pour moi. [...] Tout le temps qu'elle jouit en pleurant en criant, elle tint mon sexe comme on tient une main. Je sentais le sperme battre, juste avant de jaillir, dans sa paume comme aurait battu son sang (p. 9).

Ce «cliché " démontre une fois de plus que l'inconscient se répète incessamment, ad nauseam, parce qu'il est soumis à un déterminisme absolu, qu'il radote pour ainsi dire; pour me citer moi-même :

L'inconscient (aussi balourd et épais que subtil et raffiné dans ses moyens d'expression) est constamment répétitif, il dit la même chose cent fois plutôt qu'une parce qu'il tient absolument à être entendu, sachant (le malin) qu'on l'écoute et le comprend peu au niveau du conscient.

Une deuxième mention explicite de la masturbation :

Philomène a rencontré Bouboule. Bouboule a tassé Philomène dans un coin. Philomène s'est esquivée. [...]

...Philomène lui a dit à Bouboule que Ti-Jean je l'aime. Elle lui a joué la comédie-prétexte de la femme fidèle... Pour Philomène, il s'agit de ne pas perdre la face...

Mais Bouboule a l'habitude. II a bien vu que Philomène avait une peur bleue de son Ti-Jean. II était bandé, Bouboule, mais lui aussi a eu un peu peur de ce Ti-Jean. [...]

Et Bouboule bandé s'est crossé. [P. 25. C'est moi qui souligne.]

Le "mais Bouboule a l'habitude" surgit dans le texte comme un cheveu sur la soupe, comme un cil dans l'œil. Cela ressemble à un corps tout à fait étranger par rapport à l'enchaînement logique des idées. A moins qu'il ne s'agisse d'un simple déplacement (mécanisme inconscient) d'une idée parfaitement justifiée à propos d'autre chose et qui exigeait d'être exprimée tout autant qu'allait l'être "l'autre chose"? Ce qui s'appelle "association libre" est un paradoxe ironique et ce genre d'association relève toujours d'un déterminisme absolu! De quoi Bouboule pourrait-il bien avoir l'habitude? De la peur de Philomène? C'est la première fois qu'elle va chez ce Bouboule, pour rendre service à Berthe... Etc. Et qu'estce que c'est que le choix particulier de ce désignateur, Bouboule, dans un tel contexte? Bien sûr, de «quelqu'un qui en fait le trafic, des gouffebâles" (p. 24) : balls, mot anglais qui se traduit par «boules, balles " et ici par "boulettes " de drogue. Mais l'autre sens "vulgaire " inéluctable? II y a deux «boules" dans Bouboule (boule + boule $=$ Bouboule, comme dans loup + loup $=$ Loulou). J'exagère? Retournons donc encore au texte. 
J'aurais pas tué Bouboule si Philomène m'aurait pas tenu par les gosses. [P. 73. II y a un erratum dans la présente édition, «j'aurais tué ", que je corrige d'après l'édition de 1968. C'est moi qui souligne.]

Pareille collocation constitue évidemment une définition. Quant à ce verbe qu'utilise Renaud pour décrire l'action de Bouboule, plusieurs lectrices m'ont affirmé n'en pas connaître le sens et - naïveté, de leur part ou de la mienne? - je leur ai expliqué que le mot provenait peut-être d'une déformation de "se caresser" et, incertain de toute étymologie, je leur en ai donné le synonyme scientifique "poli "; voici, sous toutes réserves, ce qu'en dit Lapointe:

Se crosser. Le mot le plus répandu [i.e., pour parler de la masturbation]. Crosser vient du latin crottiare qui veut dire branler et secouer, courber, remuer, gauler. Une autre possibilité serait que le verbe soit formé à partir du substantif crosse qui viendrait luimême du substantif krukkjo (béquille); crosser signifierait donc chasser avec une crosse, battre. $\AA$ rapprocher de gaule.

Se gauler. Cela vient de gaule, sorte de perche ou de bâton employé à des fins multiples. Entre autres choses, on s'en sert pour secouer les arbres fruitiers ${ }^{10}$.

"Crosse/gaule/perche/bâton », cela nous garde dans la sphère du «s'faire " et de la stick-o-manie.

Le lecteur un peu attentif remarquera l'enchaînement des macroséquences: (a) "On leur arrache un petit cinq [...] y s'contentent de la main» (p. 17); (b) "Philomène s'est trouvée une djobbe comme empaqueteuse dans une manufacture de cigares. Elle met cinq gros cigares dans une boîte (etc.)" (p. 24); (c) «Et Bouboule bandé s'est crossé» (p. 25); (d) “Philomène se couche et s'endort... Une volk's... Bouboule... Cinq cigares dans une boîte... Berthe... Habon... Habon... Philomène se retourne dans son lit en s'étirant les jambes... » (p. 26). Ce rêve très court de Philomène est identique au rêve très court de "la rêveuse au frigidaire" (rapporté et interprété dans la première tranche de cet essai). Au niveau du contenu manifeste, construction du rêve sur des restes diurnes/résidus de la veille ou, si l'on veut, sur des événements récents de la réalité extérieure: Philomène a été accueillie dans une «volkswageune», elle a rendu visite à Bouboule, elle a trouvé un emploi dans une manufacture de cigares où son genre de travail l'oblige à des gestes répétitifs fastidieux, elle «a pris rendez-vous avec Berthe dans un restaurant». Mais ici, comme à propos du rêve au frigidaire, le même commentaire s'impose :

Voilà pour les éléments de la réalité extérieure insérés dans ce rêve. Matériaux objectifs du seul contenu manifeste, ils ne sauraient fournir en eux-mêmes de sens intelligible cohérent car ils ont été récupérés par l'imaginaire de la rêveuse et redonnés tels qu'elle les ressentait, elle, à travers sa propre subjectivité [...]. Mais que comprendre de cette production imaginaire fabriquée avec des morceaux de la réalité, quel sens attribuer à ces restes diurnes? 
Au niveau du contenu latent, l'interprétation doit tenir compte de ce qui précède le rêve. Philomène a été tassée dans un coin par Bouboule et si elle s'est esquivée ce n'est pas faute d'envie, c'est parce que «elle veut être prudente. Elle ne connaît pas Bouboule. [...] Peut-être parce que ça pourrait déplaire à Berthe. [...] Philomène avait une peur bleue de son Ti-Jean » (p. 25). Elle quitte donc Bouboule avec șon envie sexuelle insatisfaite et un regret clairement exprimé :

Philomène se disait en rentrant chez Louise, qu'après tout, Bouboule ou pas Bouboule, un de plus ou de moins... (p. 26)

Deuxième occasion d'exaspérer cette envie sexuelle :

Louise dormait, fatiguée.

II y a des rouleuses écrasées dans le cendrier de verre à côté du lit. Philomène ne fume pas et les cendriers l'indiffèrent. [...]

[Insertion du rêve déjà cité.]

En se levant, Louise a vidé le cendrier...

- Maudit! y font eksiprès... (P. 26.)

L'épisode des «rouleuses» dans le cendrier est un peu énigmatique pour le lecteur mais le sens de ce détail n'a pas échappé a Philomène, Ti-Jean est passé par là! C'est le texte qui le dit, avant et après ce passage cité :

Louise travaille de nuit. [...] Elle revient du travail quand Philomène part, le matin.

Ti-Jean arrive souvent après le départ de Philomène. II tasse Louise dans un coin. Louise se laisse faire. (P. 24.)

[Philomène] a recommencé à travailler dans une manufacture de cigares. Ça paye un peu, le tabac. Chaque fois que Ti-Jean s'en roule une, il pense à Philomène. Mais Philomène travaille dans les cigares. Lui, des cigares, y peut pas s'en payer. [...]

II faut rouler. Les tout-faites, c'est trop cher. [P. 31. C'est moi qui souligne.]

Le rêve de Philomène succède donc à une envie sexuelle insatisfaite et à un dépit évident du fait que Louise et Ti-Jean se sont «roulés» dans le lit où elle-même doit se coucher, dormir et rêver ( "Maudit! y font eksiprès... »), alors qu'elle-même a éconduit Bouboule et qu' «elle lui a joué la comédie-prétexte de la femme fidèle..." (p. 25) : critère du contexte sexuel pour le repérage des éléments inconscients dans le contenu manifeste et pour l'orientation de leur interprétation. Reprenons maintenant chacun des éléments de ce contenu manifeste pour tenter d'en saisir le sens subjectif au niveau du contenu latent. Une Volk's : c'est justement là que Philomène s'est remémorée que les automobiles lui sont une occasion de faire un peu d'argent en y masturbant des hommes, que «Ti-Jean avait pris sa petite main dans sa grosse patte. Tu viens avec moé Ménène? On va s'en passer une? " et que "c'te nuit-là, Ti-Jean était venu trois fois" (p. 19), c'est justement là aussi que s'ébauchent les «jeux de mains" entre Berthe et Philomène; volk's = automobile, à mettre en relation avec le passage analysé dans la première tranche, "l'automobile, mon vieux, l'automobile, c'est ça l'amour" (p. 53). Bouboule: n'oublions pas la double référence du texte à ce personnage, "et Bouboule bandé s'est crossé " (16 lignes seu- 
lement avant l'allusion du rêve à Bouboule) et «après tout, Bouboule ou pas Bouboule, un de plus ou de moins" (6 lignes seulement avant l'allusion du rêve à Bouboule); on m'objectera peut-être que Philomène ne sait rien de la masturbation de Bouboule, mais ne soyons pas trop naifs! II y a bien quelque part un sujet écrivant qui écrit le texte, qui choisit des mots et des motifs plutôt que d'autres, qui fait parler et rêver des personnages au moins partiellement imaginaires, qui leur prête parfois ses propres paroles et fantasmes, qui connaît (consciemment ou inconsciemment) d'avance l' «histoire" et ce que fera chacun de ses personnages en les/s'y confondant parfois légèrement sans s'en rendre compte; ici, concluons donc le plus simplement du monde à une irruption banalement inintentionnelle du narrateur dans le rêve dè Philomène, à une méprise inconsciente ou à un lapsus d'auteur. Cinq : inutile d'insister à nouveau. Boîte :

A la suite de ses multiples observations, Freud énumère toute une série d'objets (et ce ne sont pas les seuls), dont les BOÎTES, les vases et les objets creux en général, qui peuvent être symboliquement substitués aux organes sexuels ou génitaux de la femme ou à la femme tout entière:

Les boîtes, les coffrets, les caisses, les armoires, les poêles représentent le corps de la femme, ainsi que les cavernes, les navires et toutes les espèces de vases ${ }^{11}$.

La justification de ce sens du symbole est simple. Globalement, les organes sexuels et génitaux féminins sont formés d'une double cavité, d'une part le vagin comme réceptacle du pénis et du liquide spermatique, de l'autre l'utérus comme réceptacle du foetus et des eaux amniotiques. Cette caractéristique, la cavité, constitue ainsi le "facteur commun, le tertium comparationis" entre le symbolisé en question et le symbolisant ${ }^{12}$.

Si on passe par la série des équations ou, pour ainsi dire, des synonymes symboliques, on retrouvera dans le domaine linguistique la même association entre les organes génito-sexuels féminins et les objets creux. C'est ainsi que, dans le Pidgin-English des aborigènes de la Nouvelle-Guinée, le mot "bokis" (déformation du mot anglais «box") signifie à la fois boîte et organes génitaux féminins. Le slang américain connaît bon nombre de sobriquets appliqués au vagin, «bag, basket, can, fish pond, HOT BOX». La même association se retrouve dans le délire d'une malade mentale effrayée sans raison apparente par le bain turc qu'elle appelait «hot-box» et qui représentait pour elle un utérus ${ }^{13}$.

Mais, encore une fois, puisque c'est le texte seul qui a raison et lui seulement, retournons à notre texte voir si ces remarques générales s'y actualisent concrètement dans une individualité unique et singulière créée par Jacques Renaud, s'il s'y trouve des indices sûrs pour passer d'une simple possibilité théorique à une certitude raisonnable et attribuer ici même, dans ce rêve littéraire particulier, le sens symbolique connu à l'élément «boîte» :

Ça fait un bon bout de temps qu'il [Ti-Jean] lui a pas rabattu son gros battoir sur la tempe dans un mouvement d'impatience parce qu'un gars sur la rue avait regardé Philomene de la boîte aux tétons... [P. 33. C'est moi qui souligne et qui ajoute les caractères gras. 
Berthe. Hé! C'est d'valeur qu'a soye de même... C'est une maudite belle boîte... [P. 66. Même remarque.]

Non! Mais c'était d'voir la face à Berthe! [...] Calvasse de pelote!...

$[\ldots]$

Berthe... Berthe!... Non! mais c'était d'y voir la face... Une maudite belle boîte par exemple... [P. 71. - Même remarque.]

Je rappelle que dans ces trois derniers emplois du mot «boîte" le sens sexuel s'y appréhende directement parce qu'il y est tout à fait conscient, qu'il ne s'agit donc là que d'une métaphore et non d'un symbole (selon la définition psychanalytique restrictive conventionnelle), alors que l'emploi du même élément dans le «rêve" de Philomène ne s'y comprend qu'indirectement par le détour d'une "traduction " et de comparaisons avec d'autres passages, qu'il y constitue un symbole à proprement parler puisque le sens sexuel y est totalement inconscient. Berthe : Philomène a été accueillie dans sa voiture, elle «aurait préféré un homme» (p. 17) mais elle est bientôt séduite par la conversation et la lesbienne conductrice; comme je l'ai fait remarquer plus haut, s'ébauchent alors des «jeux de mains":

Feu vert. La main de la jeune femme touche à celle de Philomène. [...]

Philomène a réagi. Elle a porté sa main gauche à son front. Elle effrite ses mèches mouillées.

[...]

La jeune femme a pour de bon saisi la main de Philomène.

Philomène s'est retournée vers la jeune femme. Elle a tenté de retirer sa main, mais sans trop de fermeté. La jeune femme n'a pas lâché. [...]

- T'as besoin d'argent...

[...]

- Je peux t'aider... Cinq... Dix...

De la main gauche, la jeune femme joue dans les mèches mouillées de Philomène. Et Philomène ne sent aucune répulsion. Elle se sent même assez bien. [...]

[...]

La volk's secoue ses pneus comme un minou mouillé ses poils.

[...]

[La jeune femme] a froissé dans sa main un dix et le bout de papier. Philomène la regardait faire sans parler. Elle lui a laissé sa main gauche. La jeune femme y a mis la boule de papier froissé. Elle a refermé la main de Philomène sur la boule. Elle l'a regardée droit dans les yeux, calme, douce, sûre d'elle-même. Comme un homme doux, pense Philomène.

- Veux-tu être ma maîtresse?

[...]

Les lèvres minces ont touché les siennes...

[P. 18-22. C'est moi qui ajoute les caractères gras.]

C'est tout cela que véhicule le seul mot Berthe dans le "rêve" de Philomène, phénomène d'une extraordinaire condensation onirique dont il faut chercher ailleurs dans le texte l'explicitation du détail (l'équivalent des associations du psychanalysant en situation clinique). Dois-je souligner 
l'identité de certains mots de cette scène («main/cinq... Dix..." et de la songerie de Philomène dans la volk's ("on leur arrache un petit cinq, un petit dix/y s'contentent de la main ") ? Dois-je insister sur la récurrence de la main gauche ( 3 fois) dans les meches mouillees ( 2 fois) et mettre ce facteur commun, mouillées, en relation avec une comparaison tout à fait curieuse et inattendue, "la volk's secoue ses pneus comme un minou mouillé ses poils "? Peut-être, mais seulement dans une note pour qu'on ne tende pas à confondre une interprétation assez bien validée avec une simple hypothèse dont la confirmation finale ne saurait être obtenue que par des comparaisons plus nombreuses avec le reste de l'œuvre de Renaud ${ }^{14}$. Habon... Habon... : reprise d'une expression de Philomène lors de sa conversation avec Berthe (2 fois, p. 19 et p. 20 ; mais voir après quoi surgit ce deuxième "ha bon"!). Si mon analyse du contenu latent est cohérente jusqu'ici, il s'imposerait d'interpréter ces «habon " au même niveau de réutilisation subjective et ambiguë. Soupirs de contentement à la suite de la gratification masturbatoire du besoin sexuel... Le narrateur n'ajoute$t$-il pas que “Philomène se retourne dans son lit en s'étirant les jambes "? Pourquoi cette précision? Superposons maintenant, comme dirait Mauron, deux rêves, celui de la rêveuse au frigidaire et celui-ci de Philomène :

Je suis sur la cinquième rue avec mon frigidaire qui dégèle. Je suis gênée. Tout le monde me regarde.

Philomène se couche et s'endort... Une volk's... Bouboule... cinq cigares dans une boîte... Berthe... habon... habon... Philomène se retourne dans son lit en s'étirant les jambes...

Légères différences au niveau du contenu manifeste, nombreux points de rencontre au niveau du contenu latent, mais absence d'une caractéristique importante dans le rêve de Philomène: on n'y retrouve pas la marque d'une intervention du Surmoi, le «je suis gênée. Tout le monde me regarde». Et pourtant, cette marque devrait y être, au moins théoriquement. D'autant plus qu'elle s'exprime d'une manière obsédante à propos de la masturbation de Ti-Jean comme nous le constaterons dans le développement intitulé «Surmoi et Stick-o-mani[e]pulation". Tous ces personnages n'étant finalement qu'une émanation de l'imaginaire de l'auteur ou qu'une certaine réalité filtrée dans sa subjectivité, «je m'emparais de la réalité et je la donnais [...] telle que je la ressentais moi " (Renaud), à Philomène devrait être attribué le même sentiment de culpabilité qu'à Ti-Jean ${ }^{15}$. Théoriquement et pratiquement, il existerait dans ce passage, dans ce "rêve", un non-dit à retracer dans un ailleurs du texte et, selon le mécanisme du déplacement, dit explicitement mais peut-être à propos d'autres choses. A titre d'hypothèse et sans prétendre à une certitude absolue, à titre aussi d'exercice pédagogique et méthodologique applicable dans d'autres situations ${ }^{16}$, vérifions dans le texte:

[...] [Philomène a peur de mal parler] [...]

La jeune femme jette de temps à autre un regard à Philomène. [...] Philomène était contrariée. Elle est maintenant mal à l'aise. La jeune femme lui jette de temps à autre un regard trop tendre. C'est pas normal, ça, pense Philomène. 


\begin{abstract}
$[\ldots]$
[La jeune femme] regarde Philomène avec insistance [...]

De la main gauche, la jeune femme joue dans les meches mouillées de Philomène. Et Philomène ne sent aucune répulsion. Elle se sent même assez bien. Mais elle voudrait pas que les passants la voient, la regardent, que quelqu'un la reconnaisse. [P. 17 à 19. C'est moi qui souligne.]
\end{abstract}

Les lèvres minces ont touché les siennes...

Philomène a soudainement pensé à Ti-Jean qui l'attend et qui va lui faire un spîtche, aux gens qui passent sur le trottoir, pressés, oui, mais qui peuvent la voir quand même, et les autos qui dépassent la volk's... Elle a reculé. [...] Elle a couru sur la rue Bernard. Personne m'a vue, j'espère... [P. 22. Même remarque.] Pour Philomène, il s'agit de ne pas perdre la face. [P. 25. Même re-
marque.]

Ti-Jean l'observe et Philomène ne le voit pas, mais se sent observée et elle est pâle...

[...] Philomène avait déjà dit à Ti-Jean qu'un homme à grosses lunettes épaisses l'avait suivie pendant deux mois sur la rue. [...] Un jour elle l'avait vu de proche cette espèce de cave, on ne pouvait pas voir ses yeux... à cause des lunettes...

[...]

Puis c'est un sexe qui apparaît [...] Le sexe retrousse la jupe foncée de Philomène [...] Le sexe sait ce qu'il veut, où il va, on dirait qu'il a des yeux [...]

[...] Les cuisses de Philomène se mouillent de jus rouge, puis c'est le corps qui rougit et le visage trop pâle devient rouge... Ti-Jean voit tout et Philomène se sent observée... Elle a honte et elle rougit, rougit... [P. 37 à 39 . Même remarque.]

Philomène se sent un peu mal à l'aise de ne pas savoir quoi dire en présence de cette fille instruite et distinguée... Philomène a tourné son regard vers la fenêtre. La fenêtre est ouverte, pour laisser pénétrer l'air, mais les persiennes brunes sont refermées, elle ne voit pas la façade des maisons de l'autre côté de la rue...

[P. 45. Même remarque.]

Dans tous ces passages, insistance sur la "gêne", sur le "regard " inquisiteur et réprobateur : sentiment de culpabilité attribué à Philomène. Même dans la dernière phrase citée, si Philomène «ne voit pas la façade des maisons de l'autre côté de la rue" cela la rassure surtout de ce qu'elle ne peut être vue de cette façade! Jeu d'autruche bien connu, se fermer les yeux pour ne pas être regardé, ne pas voir pour ne pas être vu... ${ }^{17} \mathrm{De}$ tout ce qui précède, j'explicite les critères de repérage des éléments possiblement inconscients (donc, objet propre de l'analyse psychanalytique) et de validité des interprétations: l'ensemble du contexte (comme substitut des associations d'un psychanalysant), la complémentarité symbolique («cinq/ boîte "), la double version auto-interprétante (la rencontre du symbole et de la métaphore dans le même texte), l'origine unique et unifiante des associations "libres" (l'auteur lui-même derrière/dans chacun des personnages). Après cette longue démonstration sur l'enchaînement de quelques macro-séquences, je laisse au lecteur intéressé de continuer lui-même 
l'exercice et de conclure : il s'étonnera peut-être lui-même de la cohérence interne du texte.

Troisième mention explicite de la masturbation :

Dans le coin, près de la porte, ça hurle, ça bêle. C'est l'artiste, le gars qui est journaliste, le déviargeur au long cours qui se crosse en dessous de la table. Le sculpteur qui lui vide son verre sur la poche. L'Art. [P. 50. C'est moi qui souligne.]

Quelques lignes plus bas, “Bouboule fait un faux pas. Sa main glisse vers le bas-ventre de la femme. " (Ibid.)

\section{Quatrième mention :}

Ti-Jean méprise avec hargne les gens autour de lui [...] lls n'ont pas comme lui, une idée qui les mène, une bonne obsession, compacte, palpable... Ils n'ont pas de passion... [...]

Gagne de crosseurs!

IIs dissertent, ils s'amusent.

[P. 58-59. C'est moi qui souligne et qui ajoute les caractères gras.]

Opposition, pour Ti-Jean, de la masturbation physique et de la masturbation intellectuelle, de la bonne obsession et de la dissertation, de la passion et de la métaphysique, du palpable et de l'impalpable :

II a tout simplement parfois envie de se tranquilliser un peu et de voir les autres faire de même. Quand il est tanné, c'est dans ces momentslà qu'il pense à la même chose que tout le monde : au bonheur. Mais ça lui passe. Comme à tout le monde. On oublie vite une chose impalpable. On n'a pas, tous, les loisirs nécessaires pour nager en pleine métaphysique. [P. 29. - Même remarque.]

Palpable : du latin palpo, «tâter/toucher/caresser/flatter». Qu'on se rappelle ce que j'ai dit du sens ultime de la masturbation maniaque de TiJean, “une pression de la paume pour exister". Y a-t-il si grande différence entre Ti-Jean et «tout le monde" ? Collocation réitérée de Ti-Jean et “les gens", comme ici, comme à la page 22 ("Philomène a soudainement pensé à Ti-Jean qui l'attend et qui va lui faire un spîtche, aux gens qui passent...»). Ils s'amusent: qu'on se rappelle aussi ce que cela signifie au niveau de l'inconscient ${ }^{18}$.

Cinquième et dernière mention explicite de la masturbation dans «le Cassé »:

[...] A l'école, le maître y nous en parlait de militer... Lui y'était dans l'Église militante... [...] On se fiait à l'évangile, [...] on faisait comme Jésus-Christ disait de faire, on s'crossait pas [...]

$[\ldots]$

J'me sus tanné en calvaire. J'ai colissé ça là. [...] J'ai dit à JésusChrist d'manger d'la marde longue de même pis j'ai commencé à m'crosser en pensant à Marie-Madeleine... [...]

$[, .$.

Était comme Marylin Monroe, Marie-Madeleine ${ }^{19}$ [...] [P. 74-75. C'est moi qui souligne.]

Voilà... 
Considéré isolément, chacun des éléments de la vérification de l'hypothèse de départ risque de paraître assez léger en lui-même; ce qui pèse véritablement, c'est l'accumulation convergente des données du texte, leur réunion brin par brin dans des faisceaux homogènes.

II appartiendrait maintenant à l'histoire littéraire de retracer l'origine, l'évolution et le traitement de ce thème tabou de la masturbation dans notre littérature ${ }^{20}$. Tel qu'il se présente dans "le Cassé ", ce thème constitue une transgression des canons littéraires québécois et des valeurs religieuses québécoises de l'époque, 1964. II y a là un type de subversion littéraire et sociale dont la portée reste à évaluer. C'est la marque de l'affaiblissement de la répression de tout ce qui, de près ou de loin, touche au sexuel, répression qui avait entraîné dans une partie de la population du Québec un refoulement excessif et généralisé non seulement de la curiosité sexuelle (ce qui va de soi) mais encore de tout ce qui en dérive par déplacement et sublimation (ce qui ne va pas de soi) dont, en particulier, la curiosité intellectuelle sous toutes les formes et dans tous les domaines: théologique, philosophique, scientifique, social, politique évidemment, et même artistique. L'époque n'est pas si lointaine où, à Montréal, avait été décrété l'ultime critère de la censure, "tout ce qui bouge est indécent", et où il était mal vu de bouger, autant de la tête et des idées que des seins et du bassin. Quinze ans ont passé depuis 1964 et bien des choses ont changé de l'extérieur. Mais de l'intérieur, dans le substrat affectif, dans le Surmoi du groupe, dans ce qui se perpétue et se reproduit culturellement au niveau de la censure inconsciente? Deux indications à propos du "Cassé ". L'indignation des notables de Tracy à la mise au programme de cette nouvelle, “entre les mains des enfants de CÉGEP» (p. 188, c'est moi qui ajoute les caractères gras et qui souligne le mot «enfants») : mais il y a longtemps de cela, c'était en 1971! ’̀ l'occasion d'un cours sur l'approche psychanalytique du phénomène littéraire, j'avais choisi de préparer tranquillement, indirectement, les esprits des enfants d'UNIVERSI$T E ́$ - où situer les limites du ridicule? - en interprétant un dessin (du genre "draw-a-person test») qui traitait symboliquement du thème de la masturbation (les mains et le cinq); indignation suprême, tollé général, je ne sais comment je m'en serais sorti n'eût été du secours inattendu d'un des plus indignés qui, dans une incommensurable naïveté, affirma ceci : «A supposer que ce que vous dites soit vrai, que la main et le cinq réfèrent en tant que symboles à la masturbation, ça ne peut pas s'appliquer aux femmes." Double ironie du sort, d'une part, ce dessin avait été exécuté par une femme; d'autre part, ce sont les étudiantes qui détournèrent de ma personne l'agressivité de la classe pour la reporter sur ce pauvre naîf en le fustigeant d'un regard méprisant et en s'écriant d'un commun accord: “Comment penses-tu que les femmes se masturbent, avec leurs oreilles?" Mais il n'y a pas longtemps de cela, c'était en 1978! Indignation, réaction émotive et souvent irrationnelle. Savaient-ils seulement pourquoi, de quoi, ils s'indignaient les notables de 1971 et les étudiants de 1978? Parmi ces derniers, il s'en trouvait certains pour être en lutte contre 
"les appareils de reproduction de l'idéologie dominante"; se rendaient-ils compte, eux qui contestent si facilement l'existence de l'Inconscient, qu'ils reproduisaient eux-mêmes et inconsciemment les effets réprimés et refoulés d'une idéologie "dominante»? Conclusions: sourire (ou pleurer?) et promouvoir l'Inconscient au rang des "appareils reproducteurs", à la place d'honneur attribuable au plus efficace, au plus insidieux, donc au plus dangereux de tous; laisser aux authentiques créateurs leur plus entière liberté pour leur permettre de remplir pleinement un rôle socio-culturel indispensable, celui de la transgression et de la subversion de normes et de valeurs usées ${ }^{21}$.

(A SUIVRE)

Henri-Paul Jacques, Université du Québec à Montréal

1. La première tranche de cet essai a été publiée dans la précédente livraison de Voix et images, vol. IV, $\mathrm{n}^{\circ} 1$. - Les passages cités, sans autres références que la pagination, seront extraits de la dernière édition du "Cassé": Jacques Renaud, le Cassé et autres nouvelles, suivi de le Journal du CASSÉ (1964), Montréal, Éditions Parti pris, 1977 ( $3^{\mathrm{e}}$ édition revue et augmentée).

2. L'inconscient trouve toujours, dans une langue ou l'autre et souvent dans plusieurs langues non apparentées à leurs origines, les coïncidences sémantiques nécessaires à l'expression de ses "vérités " les plus fondamentales. Ainsi, le mot verge («baguette/membre viril") reproduit exactement le double sens de son "etymon " latin virga; de même, en anglais, le même double sens des mots yard et rod (également dans son "etymon" germanique Rute), etc. Si ces coïncidences sémantiques se créent partout de cette manière, cela est dû, d'une part, au phénomène de l' "analogon »/ “tertium comparationis »/facteur commun; d'autre part, à la rencontre des deux codes (celui de l'inconscient et celui du conscient). II ne faut jamais oublier cette caractéristique essentielie du symbole, tel que défini en psychanalyse freudienne : il n'y a symbole que si le sens sexuel du mot utilisé par le sujet est totalement inconscient, autrement il s'agit d'une simple "métaphore" (pour préciser un point de méthode, notons que c'est par comparaison à ce type de métaphores sexuelles qu'il est le plus facile d'établir le sens sexuel inconscient d'un symbole). Pour ces coïncidences particulières, voir: A. Ernout et A. Meillet, Dictionnaire étymologique de la langue latine (1932), Paris, Klincksieck, 1959 ( $4^{e}$ édition revue, corrigée et augmentée), s.v. virga; Theodore Thass-Thienemann, The Interpretation of Language, Volume II : Understanding the Unconscious Meaning of Language (1968), New York, Jason Aronson, 1973, p. 101. Pour le problème général de ce type de coïncidences, voir : $\mathrm{S}$. Freud, l'Interprétation des rêves (1900), Paris, P.U.F., 1973, p. 302 ("Ce qui est lié symboliquement fut vraisemblablement lié autrefois par une identité conceptuelle et linguistique. Le rapport symbolique paraît être un reste et une marque d'identité ancienne. On peut remarquer à ce propos que dans toute une série de cas la communauté de symbole va bien au-delà de la communauté linguistique »); du même auteur, Introduction à la psychanalyse (1916), Paris, Payot ("pbp" $\mathrm{n}^{\circ}$ 6), 1974, p. 84 ("Le langage, qui ne doit rien au hasard, mais constitue pour ainsi dire la cristallisation des connaissances accumulées, le langage 
[...] qu'on ne doit cependant pas utiliser sans précautions $\%$ ) et tout le chapitre 10 sur "le Symbolisme dans le rêve"; Henri-Paul Jacques, Mythologie et psychanalyse: le Châtiment des Danaĩdes, Montréal, Leméac, 1969, où je traite longuement de ce sujet, p. 37 , note 39 et passim.

3. C'est en faisant du pouce (un des «doigts" de la main) que "Philomène a déjà perdu ben du temps pis sa cerisen (p. 16). Avant, elle était "vierge", en latin virgo ( «jeune fille ou jeune femme qui n'a pas encore connu l'homme»). "Vierge/verge" et virgo/virga; virgo, c'est-à-dire “qui n'a pas encore connu la virga ", "homme " se disant d'ailleurs vir en latin tout comme l' "hommâtre ", la femme hommasse, se dit virago dans la même langue. Je reviendrai sur les rapports de l'inconscient et de l'étymologie (objectivement vraie ou fausse, ce qui n'a aucune importance pour l'inconscient); pour ceux qui voudraient approfondir ces fantasmes universels, ce que j'appelle les universaux de l'imaginaire, autour du monde végétal, "bâton/cerise", je les renvoie a Thass-Thienemann, op. cit., p. 100-103, «Tree and Man".

4. Je donne au mot collocation son sens étymologique latin, «réunion en un même lieu d'éléments différents". Cette étiquette décrit autre chose que la simple juxtaposition. Dans un passage du "Cassé ": "Les filles. Et plus loin encore, plus loin, jusqu'à Percé. Les Gaspésiennes [...]" (p. 14), il y a juxtaposition de "Percé/Gaspésiennes» mais collocation des trois éléments «filles [...]/Percé/Gaspésiennes " : la juxtaposition est une collocation immédiate qui exclut toute séparation ou écart entre deux éléments. On constatera l'utilité de cette distinction dans la suite de cette analyse, en particulier lorsqu'il sera question de générativité verbale et textuelle.

5. "Y s'contentent de la main", expression éminemment ambiguë à entendre analytiquement dans les deux sens du mot, c'est-à-dire par décomposition de l'ensemble en chacune de ses unités et "psychanalytiquement " par une écoute littérale/dé-métaphorisante: (a) «ils n'en exigent/demandent pas plus» dont le sens est obvie mais usé, secondaire, figuré; (b) "ils reçoivent leur contentement/ils se font [faire] plaisir»; (c) “de», i.e. «à l'aide de/grâce à "; (d) "la " réfère à la main de Philomène mais aussi au sujet grammatical comme dans la forme pronominale absolue à l'infinitif, "se contenter [soimême] de la main". C'est exactement ce qu'exprime, et sans aucune ambiguïté cette fois, un très joli poème en prose qui présente un grand nombre de parallèles étonnants avec «le Cassé»:

" $J$ 'ai deux maudits grands bras, des bras longs comme des pissettes de beu. C'est à cause de cela que mon père m'a surnommé la pieuvre.

II a raison. Je suis une vraie pieuvre. Quand je mets la main sur quelque chose, il n'y a rien ni personne pour me l'enlever. [...] Comme la pieuvre, j'éjecte du liquide. J'ai toujours éjecté. C'est ma façon de produire. [...] Mais elle [mère], elle a accepté cet homme, elle a pris plaisir à faire l'amour avec lui; elle l'a caressé, elle m'a fait avec cet homme. Je la méprise. Depuis que j'ai compris ça, je me contente tout seul. Jamais je ne ferai d'enfants avec une femme."

[Guy Godin, IOM, Trois-RIvières, Éditions des Forges, 1971, p. 17-18. C'est moi qui ajoute les caractères gras dans la citation.]

Les mêmes mots et les mêmes fantasmes que dans "le Cassé ", y compris le fantasme d'engendrement par la masturbation, sans la femme (voir la première tranche de cet essai), et jusqu'au mot "éjecter " apparenté à "éjaculer» par l'identité de leur etymon latin (jacio, "lancer") : [Philomène] a saisi la poignée de la portière de l'auto à deux mains. Elle s'est éjectée de la volk's " (p. 22). Dans ce contexte de masturbation, et comme par hasard encore, Godin utilise lui aussi le chiffre privilégié cinq : "Un homme avec lequel elle avait fait cinq enfants par-dessus le marché." (p. 24-25). La séquence est remarquable, «caresser cet homme/(se contenter tout seul)/ faire cinq enfants".

6. L'énorme difficulté avec le «symbole", c'est celle des critères que la pratique (clinique) de la psychanalyse n'a jamais explicités, pas plus pour les profanes que pour les spécialistes, sauf pour dire que l'analysant se trouve incapable 
de produire des associations personnelles à partir d'un élément symbolique. C'est pour cette raison que je répète constamment que «tout n'est pas symbole et il faut sans cesse et toujours rappeler la plaisanterie traditionnelle chez les psychanalystes :

L'avion est un symbole sexuel, mais qui sert aussi à voler de Vienne à Munich.

L'avion est un symbole sexuel, mais qui sert aussi à voler de Vienne à Munich.

L'AVION EST UN SYMBOLE SEXUEL, MAIS QUI SERT AUSSI A VOLER

DE VIENNE A MUNICH». [Voir la première tranche de cet essai.]

Voilà pourquoi j'insiste sur ce critère du «contexte sexuel » dans l'application de la psychanalyse et de l'interprétation symbolique à la littérature. Tout récipient PEUT être un symbole de la femme et de ses organes génito-sexuels en raison de l'analogon, mais personne au monde ne me fera avaler cette énormité d'une interprétation "symbolique " de la fable de "Perrette et le pot au lait": La Fontaine a désexualisé le contexte du pot alors que dans le textetuteur (récits indiens datant de 500 de notre ère) le pot y est évidemment un symbole dans un contexte explicitement sexuel (voir le Chatiment des Danaides, p. 101-102).

7. S. Freud, l'interprétation des rêves, p. 333 (voir également p. 229); on pourra comparer avec ce qui est dit dans son article "Sur les souvenirs-écrans" (1899) à la page 129 dans Névrose, psychose et perversion, Paris, P.U.F., 1973, p. 113-132.

8. Voir Ernout-Meillet, op. cit., s.v., et Thass-Thienemann, op. cit., p. 52. Quand Freud écrit «je ne saurais dire d'où viennent ces expressions, quelle image est au fond ", il en oublie lui-même sa psychanalyse : l'effet observable de l'inconscient pourrait-il jamais être autre chose qu'une «formation de compromis " qui véhicule simultanément, d'une manière indissociable, l'effet combiné de la pulsion et de la défense (Ça+Moi) ? A partir uniquement de l'analyse des fantasmes exprimés dans le matériel linguistique. Thass-Thienemann arrive à cette conclusion sur ce qui est resté énigmatique pour Freud en 1900 :

The discovery of the autoerotic secrets of the self coincides with the time of sex recognition; these two moments, therefore, are closely associated with one another. The feeling of anxiety and guilt which pervades the one permeates the other too. The frustration inherent in sex in all higher culture enters the infant at an early age. In a more explicit expression : Masturbation and sex recognition are both inherently associated with castration fantasies. (op. cit., p. 68-68.)

Je ne traduirai que la phrase essentielle, celle que l'auteur a lui-même soulignée: "La masturbation et la reconnaissance des différences sexuelles sont intimement associées à des fantasmes de castration. " Nous en aurons une manifestation concrète dans ele Cassé " quand j'aborderai la se[x]ction (autre preuve sémantique de ce qui est ici affirmé, "sexe " et "sectionner» dérivant du même etymon latin seco, "couper ") que j'ai intitulée "le Surmoi et la sticko-manile]pulation ».

9. Marc Cholodenko, le Roi des fées (1974), Paris, U.G.E., «10/18», n 1166, 1977 , p. 83-84. - Cette citation me permet d'attirer l'attention sur un phénomène curieux et extrêmement fréquent : «ma main/maintenant/tint/comme on tient une main " où les formes «tint/tient " appartiennent au même paradigme que "tenant"; in masturbationis situ, décomposition de l'ensemble «maintenant " qui signifie "tenir de la main" dans le code de l'inconscient, et réutilisation des deux éléments par collocation. Aux incrédules dont cette remarque choquerait la logique consciente je recommande de constater le fonctionnement de ce phénomène dans les trois textes cités ("le Cassé», IOM, le Roi des fées) en dressant une liste écrite des nombreuses occurrences: leur incrédulité et leur surprise n'en seront que plus grandes! Ils pourront trouver une ébauche d'explication de ce «maintenant» dans Thass-Thienemann (op. cit., p. 64) ainsi que de ma glose sur "une pression de la paume pour exister "(dans la première tranche de cet essai) : Cholodenko utilise aussi le même 
mot «paume" dans un même contexte. Ce n'est pas par hasard que j'ai parlé de la "masturbation maniaque " de Ti-Jean et de "stick-o-mani[e]pulation " (où doivent se lire les mots "manie " et "manipulation", celui-cl étant formé dans sa première partie de l' "etymon " latin manus/ "main ") : l'idée obsédante de la main se manifeste dans le texte jusque dans le choix de mots homophones et de mots véritablement ou faussement dérivés de MAN-us, comme dans "mancuvre", "manufacture" (fabrication à la main), "maniaque " (dont l'élément man- n'a de rapport que phonique avec man-us), etc. Qu'il me suffise, sans plus, de mentionner l'existence de ce phénomène dont une analyse plus poussée m'entraînerait trop loin.

10. Ghislain Lapointe, les Mamelles de ma grand-mere - Les Mamelles de mon grand-frère, Ville Saint-Laurent, Éditions québécoises, 1974, p. 53. J'ai utilisé le mot «poli» (entre guillemets) pour rappeler une autre expression également relevée par Lapointe, "se polir le shaft", et le «frottement" que j'ai glosé dans la première tranche de cet essai : le "polissage " s'obtient toujours par "frottement". Cohérence de la langue et de l'inconscient. Je saisis l'occasion que me donne cette citation pour illustrer encore une fois le phénomène de la juxtaposition «innocente» de toutes sortes d'expressions toutes faites et de clichés usés : "Se gauler. Cela vient [...]", évidemment!

11. S. Freud, l'Interprétation des rêves, p. 304; également dans Introduction à la psychanalyse, p. 146 et p. 141.

12. En effet, pour Freud, "l'essence du rapport symbolique consiste dans une comparaison" et pour justifier un symbole, il faut "chercher le facteur commun, le tertium comparationis de la comparaison présumée". (Ibid., p. 137.)

13. Ces remarques sont, à quelques modifications près, extraites de le Chátiment des Danaildes, p. 80-81 et 107-108.

14. "L'opposition droite/gauche s'avère équivaloir à bien/mal dans presque toutes nos langues»: Theodore Thass-Thienemann, The Interpretation of Language, Volume 1: Understanding the Symbolic Meaning of Language (1968), New York, Jason Aronson, 1973, p. 267 (la démonstration détaillée en est donnée p. 266-271; ainsi que dans son Volume // déjà cité, p. 85-86 et 200). La même conception s'est également cristallisée dans les superstitions et dictons de la plupart des nations (par ex. : Albert K. Weinberg, «The Psychological Analysis of Superstition ", The Psychoanalytic Review, vol. 7, 1920, p. 31-42) et se retrouve dans la symbolique onirique et générale: « La gauche peut signifier l'inceste, l'homosexualité, la perversion, alors que la droite signifie le mariage, etc. " , selon Wilhelm Stekel, The Language of Dreams (1911), Boston, Richard G. Badger, 1922, p. 213 (exemples à l'appui, p. 213-226). Pour une étude plus récente et qui fait appel aux recherches neurologiques, voir Jeannine Herron, "Les gauchers ont-ils un cerveau différent?", Psychologie, $n^{\circ} 77$, juin 1976, p. 25-28.

Ainsi, à analyser chacun des éléments de cette série située dans un contexte d'allusions à la masturbation, on obtient ceci : "main-gauche-mèches mouillées-minou mouillé". Les "mèches" et le "minou" sont liés entre eux par deux facteurs communs dans le texte, "poils" et “mouillées/mouillé". Quant au «minou», le sens métaphorique grivois est bien connu, "chat/chatte/ chaton/pussycat/etc." Cette coïncidence linguistique (français/anglais) qui mime la coïncidence métaphore/symbole (conscient/inconscient) est loin d'être isolée; un seul exemple parmi bien d'autres :

Quand I'homme blanc s'établit dans les îles de la Nouvelle-Zélande [...] il apporta avec lui le chat domestique européen. Avant son arrivée, les aborigènes maoris n'avaient jamais eu connaissance de l'existence du chat. lls devaient donc lui trouver un nom et ils choisirent le mot tori ou tore. Or ce mot existait déjà dans la langue des Maoris : tore désignait les organes génito-sexuels féminins, [...et] kiritore les petites lèvres.

(Sidney J. Baker, "Language and Dreams", The International Journal of Psycho-Analysis, vol. 31, 1950, p. 171-178: p .171 pour la citation.) 
Comme pour la «boite», le «minou» se retrouve ailleurs dans le texte: “... Mémène, viens ma Mémène, viens mon p’tit minou, viens.» (p. 54). Celui qui n'a pas l'habitude des voi[es]x d'expression de l'inconscient manifestera d'énormes réticences aux mises en rapport de ces "morceaux" de texte, d'où la nécessité de répéter les critères de repérage et d'interprétation: le contexte explicitement sexuel, la singularité de la comparaison, le lien par les facteurs communs, l'importance considérable de l'automobile dans l'imaginaire de l'auteur. On se rendra vite compte de cette importance à relever et comparer tous les passages qui se rapportent à l'automobile dans "le Cassé"; la superposition des trois passages suivants suffira : “L'automobile, mon vieux, l'automobile, c'est ça l'amour » (p. 53)/ «des pneus qui font un bruit de langue sur l'asphalte, un son de bouche mouillée ininterrompu " (p. 14)/ «la volk's secoue ses pneus comme un minou mouille ses poils" (p. 19). L'automobile réfère à l'animé, au corps humain, à l'amour! Indépendamment de l'interprétation sexuelle donnée jusqu'ici à de tels éléments du texte. je fais la gageure que l'automobile a représenté dans l'enfance de Ti-Jean/Renaud un irremplaçable et terrible "objet transitionnel" (Winnicott, voir s.v. dans le Vocabulaire de la psychanalyse):

Les totos. Les bébés. Ça se ressemble. C'est comme ça qu'on appelait ça, quand j'étais petit, des totos. Je les faisais rouler sur les dessins du tapis. [...]

C'est drôle comme les criards des totos ça me fait penser à des cris de bébés. [P. 76, cf. également p. 72.]

J'espère pouvoir revenir plus en détails sur ce sujet qui constitue un des liens essentiels de la cohérence interne du «Cassé».

15. II arrive souvent que, par décomposition (mécanisme inverse de la condensation), les personnages d'un rêve représentent des aspects différents de la personnalité du rêveur, de ses désirs, de sa propre censure surmoiïque; je pense à tel film de Belmondo (dont j'oublie le titre) où, dans un cauchemar, Belmondo jouait tous les rôles, juge, accusé, avocat de la Couronne et avocat de la défense, témoins à charge et à décharge, policiers, greffier, etc. Mutatis mutandis, le même phénomène ne peut pas ne pas jouer à l'occasion dans les projections littéraires d'un écrivain. Si ce phénomène s'applique ici, il faudrait en conclure à des déplacements et à des dédoublements de personnages pour la masturbation (Bouboule/Philomène/Ti-Jean/...) et à une possibilité de transposition littéraire pour l'épisode homosexuel féminin (Philomène/Berthe)...

16. Ce type de déplacement est fréquent, j'en ai relevé un autre cas plus haut: "Mais Bouboule a l'habitude. [...] Et Bouboule bandé s'est crossé.» (p. 25). Un autre exemple difficilement contestable tiré d'Anne Hébert, "le Torrent" (1945), p. 9-46, in le Torrent, Montréal, Éditions H.M.H., 1976. Dans son désir de contempler une face humaine, François le bâtard est allé se poster au bord de la grand'route et il y rencontre un ivrogne mais sa mère survient bientôt :

L'homme, surpris, se leva péniblement. II semblait fasciné par ma mère autant que je l'ètais. [...]

[...] L'homme parlait à ma mère. I" paraissait la connaître. II disait de sa voix traînante:

- Si c'est pas la belle Claudine!... Te retrouver ici!... T'as quitté le village à cause du petit, hein? [P. $14-C^{\prime}$ est moi qui souligne.]

Les phrases soulignées constituent en elles-mêmes un indice; mais la confirmation de l'indice se trouve un peu plus haut, par déplacement, dans la description de l'approche de Claudine: "Dans sa main elle tenait la maitresse branche qui servait à faire rentrer les vaches." (Ibid.) La présence de cet adjectif est absolument bizarre; bien sûr, la grande Claudine a besoin d'un bâton "pour frapper l'homme à la tête" (p. 15), mais c'est avec une "hart" et non avec une "trique" (p. 15) qu'on fait rentrer les vaches! A travers un cliché encore apparemment innocent, "aveu » involontaire du texte qui révèle l'identité du père de François le bâtard. 
17. Ce rêve de Philomène et le cauchemar de Ti-Jean (partiellement analysé dans la première tranche) miment d'une manière extraordinairement fidèle les rêves et les cauchemars les plus authentiques, d'où la légitimité de les interpréter comme tels. - Une indication du texte à l'appui de l'interprétation sexuelle du rêve de Philomène et du cauchemar de Ti-Jean: "Ti-Jean s'est mis à penser aux gens couchés, ceux qui dormaient et ceux qui ne dormaient pas. Ceux qui rêvent après leurs pamoisons de corps, poussés à bout de fatigue comme les locomotives dans les pentes. " (p. 72). Sur ce sujet, les études tant expérimentales que cliniques sont nombreuses et la critique littéraire peut $y$ prendre beaucoup (analogies entre la transposition onirique et la transposition littéraire, traduction symbolique du texte " $\mathrm{cru}$ ", etc.) : Victor W. Eisenstein, «Dreams following Intercourse". The Psychoanalytic Quarterly, vol. 18, 1949, p. 154-172; Charles Fisher, "Dreaming and Sexuality ", p. 537-569 in Psychoanalysis - A General Psychology: Essays in Honor of Heinz Hartmann, edited by R. M. Loewenstein et al., New York, I.U.P. 1966; Lawrence Stross \& Howard Shevrin, "Hypnosis as a Method for Investigating Unconscious Thought Process", Journal of the American Psychoanalytic Association, vol. 17,1969 , p. $100-135$, et la suite dans le vol. 18 ; etc.

18. Voir, dans la première tranche, la note 23.

19. Je relève ici une "curiosité" que je n'attribue pas au hasard: (a) J.-C., "Jésus-Christ/Jean le Cassé »; (b) M. M., «Marie-Madeleine/Marylin Monroe/ MéMène". C'est à une "Madeleine" que Renaud a dédié En d'autres paysages, Montréal, Éditions Parti pris, 1970; s'y retrouve un personnage important nommé «Marie" (ce qui donnerait "Marie-Madeleine"?); de même, comme ici, un Bâtard nommé “Jean-Guy" (cf. "Ti-Jean") ainsi qu'un «Luc (réversion de «Ti Cul»). Nous y reviendrons à propos du phénomène de la pluri/ multiversion et plus particulièrement à propos des comparaisons intertextuelles internes.

20. Il nous faudrait un Foucault de I'histoire de la sexualité dans la littérature québécoise. Sur le thème de la masturbation, voici quelques indications.

(a) Une timide allusion de Jean-Charles Harvey qui fait ainsi parler Dumont, le poète-débauché :

Toi, Max, ta vie est trop propre pour que tu sois complet. Tu n'as pas même eu l'opprobre du vice solitaire, car les femmes t'ont aimé avant de te donner le temps de te consumer en désirs. Tu ne connais pas cette souffrance. Et vous tous qui m'écoutez, pourquoi ces mines scandalisées?

(Les Demi-civilisés (1934), Montréal, Les Éditions de l'homme, 1962, p. 91.)

Cette référence au "vice solitaire" devrait tendre à activer dans l'inconscient du discours la référence à la “main ": il s'agit là d'une complémentarité presque obligée dont l'existence théorique résulte d'une généralisation postulée par une multitude d'observations empiriques. A partir de ce postulat et de celui de la primauté de l'image acoustique sur l'image graphique (voir les notes 29 et 30 dans la première tranche), il faut tenter d'éviter le piège de la graphie (transposition toujours artificielle et secondaire du phonique) et de repérer la «main" tout à côté du "vice solitaire". Écouter le texte après l'avoir lu: "tu n'as pas même eu». Pousser à l'extrême limite de ce qu'il permet d'entendre le principe de la primauté du phonique et récrire autrement ce qui a été entendu, en respectant intégralement l'image acoustique émise par le texte, «main mue ». Phénomène banal des évocations associatives complémentaires comme «mère/ père ":

Le visage de ma mère est beau pour rien. [...] Si on se laisse faire, ça nous désespère.

(Réjean Ducharme, l'Avalée des avalés, Paris, Gallimard, 1966, p. 7.) 
Avec plus d'évidence encore, comme dans ceci (de je ne sais plus que surréaliste) qui est bien connu: "De deux choses, l'une; l'autre, c'est le soleil.»

Soit, "l'une/l'autre" dans l'ordre du Sé et al'une- (lune)/soleil " dans l'ordre du SA où il faut remplir une case vide, expliciter un non-écrit (lune) entendu dans l'image acoustique dite par l'auteur.

(b) Une insistance assez prononcée sur ce thème dans Laurent Girouard, la Ville inhumaine, Montréal, Éditions Parti pris, 1964. Thème traité d'une façon extrêmement délicate et pénétrante, qui rejoint le sens de la masturbation dans «le Cassé " (s'agripper à son sexe pour s'agripper à la réalité et ne pas sombrer dans l'abîme de la psychose): voir les pages $13,75,80,85,109,159$. Pour ces deux auteurs (Renaud et Girouard), même éditeur, même année de publication de la nouvelle et du roman, mème cercle idéologique et littéraire («Parti pris»), rencontres nombreuses entre les deux textes (intertextualité assez évidente).

(c) Mêmes remarques pour un autre auteur de "Parti pris», André Major, la Chair de poule (1965), Montréal, Éditions Parti pris, 1973, p. 11, 62, 69-71-72, 85, 107.

(d) Réjean Ducharme dans l'Avalée des avalés, Paris, Gallimard, 1966 (comme à la page 171, sous prétexte de faire rire) et dans le Nez qui voque, Paris, Gallimard, 1967 ( «se hortenseturber»). Comme dans «le Cassé», importance des mains, des doigts, de la paume, du chiffre cinq, de la guitare, etc.

(e) Jean-Jules Richard, Faites leur boire le fleuve, Montréal, Le Cercle du Livre de France, 1970: voir p. 15, 37, 171, etc. L'ensemble associatif “cing/ main (agressante ou caressante) "se trouvait déjà comme lien d'unification des treize nouvelles publiées dans Ville rouge (1949), Montréal, Leméac, 1976.

(f) Quelques autres textes, au hasard des lectures: Emmanuel Cocke, Va voir au ciel si j'y suis, Montréal, Éditions du Jour, 1971; Jean-Paul Filion, Saint-André Avellin... le premier cóté du monde, Montréal, Leméac, 1975; Marie-Claire Blais, Une saison dans la vie d'Emmanuel, Montréal, Les Éditions Quinze, 1976.

21. Une mise en garde s'impose ici. Le but de ces remarques ne vise évidemment pas à suggérer une apologie de la masturbation, mais bien (comme on le constatera dans la suite) à analyser concrètement les conséquences d'une idéologie pseudo-médicale relativement récente (200 ans?) au sujet de la masturbation, idéologie qui n'a été reprise que tout à fait secondairement par l'éducation religieuse. 